\title{
Feasibility of Imported Self-Management Program for Elderly People with Chronic Pain: A Single-Arm Confirmatory Trial
}

Tatsunori Ikemoto - Yukiko Shiro (D) - Kayo Ikemoto •

Kazuhiro Hayashi · Young-Chang Arai - Masataka Deie ·

Lee Beeston · Bradley Wood · Michael Nicholas

Received: July 19, 2020 / Published online: August 25, 2020

(C) The Author(s) 2020

\section{ABSTRACT}

Introduction: Multidisciplinary pain management programs incorporating a cognitive-behavioral therapy (CBT) approach have been reported to be helpful for elderly people with chronic pain. However, it is unclear whether the same program for elderly people with chronic pain would translate to different cultures. This study investigated whether a multidisciplinary program based on that of Nicholas et al. (Pain 154(6):824-835, 2013) in Australia would be effective for elderly people with chronic pain in Japan.

Methods: Twenty-seven community-dwelling elderly people with chronic pain were enrolled

Digital Features To view digital features for this article go to https://doi.org/10.6084/m9.figshare.12789740.

T. Ikemoto $\cdot$ M. Deie

Department of Orthopaedics, Aichi Medical

University, Aichi, Japan

T. Ikemoto $\cdot$ L. Beeston $\cdot$ B. Wood $\cdot$ M. Nicholas Pain Management Research Institute, Faculty of Medicine and Health, University of Sydney and Royal North Shore Hospital, Sydney, NSW, Australia

Y. Shiro $(\bowtie)$

Nagoya Gakuin University, Nagoya, Japan

e-mail: shiro823@ngu.ac.jp

T. Ikemoto - K. Ikemoto · K. Hayashi · Y.-C. Arai Research of Pain Science, Non-Profit Organization, Nagoya, Japan to confirm changes (effect size $d=0.5$ ) in pain disability, which were previously reported by Nicholas et al. The multidisciplinary program consisted of eight sessions (2 sessions a week for 4 weeks). Pain disability was assessed using the Pain Disability Assessment Scale (PDAS) as the primary outcome at the baseline, the beginning and the end of the program, and the 1- and 3-month (final) follow-up. We also assessed the pain severity, catastrophizing, pain self-efficacy, and physical function with the Timed Up and Go test (TUG) and the two-step test as secondary outcomes.

Results: PDAS, pain catastrophizing, and pain self-efficacy were significantly improved immediately after the program compared with baseline, and these effects were maintained at 3-month follow-up. The effect size (d) for the PDAS score was a medium size (0.54) from baseline to 3-month follow-up. Those who showed improvements in TUG immediately after the program tended to report improved psychometric measures at 3-month follow-up.

Conclusion: These results suggest that the Japanese multidisciplinary program has a similar effect on pain disability as that reported by Nicholas et al. This finding has important implications for the development of pain services in community-dwelling elderly Japanese.

Keywords: Chronic pain; Elderly people; Selfmanagement program 


\section{Key Summary Points}

Why carry out this study?

Multidisciplinary programs in conjunction with a cognitive behavioral therapy approach have been reported to be a useful strategy for elderly people with chronic pain.

However, it is unclear whether the same program for elderly people with chronic pain would work in different cultures.

We aimed to ascertain whether the multidisciplinary program imported from another country was feasible for community-dwelling elderly people with chronic pain in Japan.

\section{What was learned from the study?}

We found that the imported multidisciplinary program was effective across cultures in improving pain disability for elderly Japanese with chronic pain.

This finding has important implications for the development of pain services in community-dwelling elderly Japanese.

\section{INTRODUCTION}

Japan is facing the advent of a "super-aged" society sooner and more rapidly than any other country in the world [1]. As a general health issue, chronic non-cancer pain is a common problem and is often associated with impairment of physical and psychological health in older people worldwide [2]. Nakai et al. reported that $42.7 \%$ of community-dwelling older adults had chronic pain, and chronic pain was associated with frailty and pre-frailty [3]. Hirase et al. reported that $60.4 \%$ of community-dwelling older adults with frailty had chronic pain, and chronic pain was associated with negative emotional and cognitive aspects of pain, as well as a decline in activities of daily living (ADL) and lower physical activity levels [4].

In recent years, there has been growing evidence that multidisciplinary pain management programs teaching self-management strategies using a cognitive behavioral therapy (CBT) approach are effective for helping people with chronic pain amongst younger and middle-aged generations as well as elderly people [5-9]. A randomized controlled trial by Nicholas et al. [5] reported a number of clinically significant improvements following their treatment program with elderly patients, including improvement in usual pain, disability, depression, and fear-avoidance beliefs. Importantly, these improvements were maintained over a 1-year follow-up period [6]. However, such CBT-based programs are rarely utilized by health professionals in Japan, and none have been reported with elderly patients in this country.

In 2017, we learned about the usefulness of the multidisciplinary program for elderly people with chronic pain in Australia, and hypothesized that the program would lead to similar results with elderly people with pain in Japan. Despite several reports showing the utility of multidisciplinary programs in younger patients with chronic pain in Japan [10-12], it was unclear whether multidisciplinary programs teaching self-management strategies were feasible for elderly people with chronic pain, because of cultural factors [13].

Therefore, in this study, we aimed to ascertain whether a multidisciplinary pain management program based on that described by Nicholas et al. [5] was feasible for communitydwelling elderly people with chronic pain in Japan, using a confirmatory case series.

\section{METHODS}

\section{Ethics}

This study was approved by the Nagoya-Gakuin University Board of Ethics (No. 2017-5). After being informed of the purpose and protocol of the study, participants provided written informed consent before taking part in the study. All procedures performed in studies 
involving human participants were in accordance with the 1964 Helsinki Declaration and its later amendments or comparable ethical standards.

\section{Sample Size Estimation and Outcome}

As this was a confirmatory study, the sample size was estimated by the assumption of changes (effect size $d=0.5$ ) in pain disability as reported by Nicholas et al. [5]. We set the improvement in pain disability as the primary outcome because it is an important focus in the treatment of chronic pain [14]. Secondary outcomes included pain intensity, pain interference, pain catastrophizing, pain self-efficacy, physical function, and adverse events.

Based on this report, at least 27 subjects were required to detect this assumption with effect size $(0.5), \alpha(0.05)$, and a power $\beta(0.8)$ in the prior analysis. In this study, we adopted a perprotocol (PP) analysis because a PP analysis measures the treatment effect only for patients who comply with the protocol [15]. In accordance with a PP model analysis, participants were recruited until 27 subjects had completed a 3 month-follow-up.

\section{Participants}

The recruitment of participants was performed by announcing the program in a local public newsletter in Seto-city, Aichi, Japan. The newsletter was published 6 weeks before the program. The inclusion criteria were as follows: (1) aged 65 years or older, (2) having pain for longer than 3 months, (4) able to attend the 180 -min sessions at the public hall in Seto-city twice weekly for 4 weeks, (5) native Japanese speaker, and (6) clearance by attending doctors for participation in light exercise and stretch program. Exclusion criteria were as follows: (1) diagnosed ongoing malignancies, (2) presence of dementia or active major mental disorder, (3) required care by others for daily living, and (4) further medical or surgical treatment for pain condition planned.

Because of limited resources, the number of participants was restricted to 10 in each program. Four programs were conducted from September 2018 to February 2020. Although 35 individuals applied to participate in the program, seven did not participate because of attendance difficulties. Twenty-eight participants completed the 4-week program: nine in the first program, seven in the second program, three in the third program, and nine in the fourth program. One participant in the first program was lost to follow-up for personal reasons (Fig. 1).

\section{Measures}

\section{Self-Report Measures}

The same set of questionnaires was completed by all participants a month prior to attending the program (baseline), at the beginning and the end of the program (first and last day), and at the 1- and 3-month follow-ups. The followup measures were performed where the program was conducted. Questionnaires included (1) the

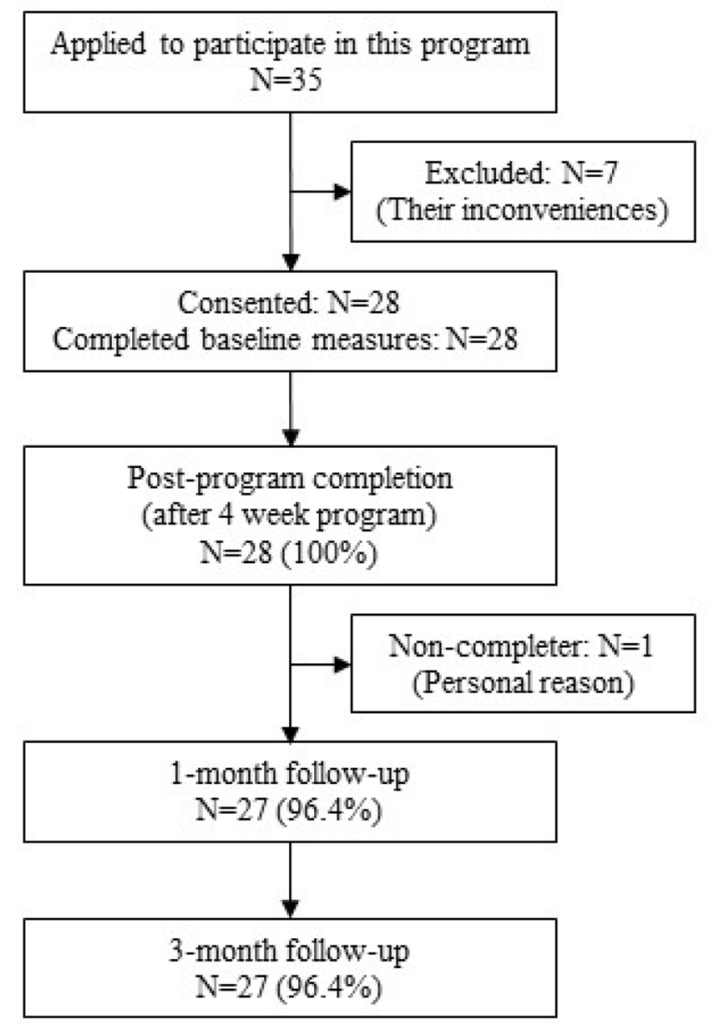

Fig. 1 Study participant flow chart 
short form of the Brief Pain Inventory (BPI) [16], (2) Pain Disability Assessment Scale (PDAS) [17], (3) six-item short form of the Pain Catastrophizing Scale (PCS-6) [18], and (4) the two-item short form of the Pain Self-Efficacy Questionnaire (PSEQ-2) [19]. All questionnaires provided were Japanese translations of the originals [20].

When patient-report outcomes are assessed, according to the Consensus-Based Standards for the Selection of Health Measurement Instruments (COSMIN), each questionnaire needs to be confirmed as a unidimensional scale. Internal consistency is the degree of the interrelatedness among items, assuming the questionnaire to be unidimensional. Cronbach's alpha is often used as a measure of internal consistency, and a value $>0.7$ is considered to be adequate [21]. We calculated these values for each questionnaire.

BPI [16] The BPI was originally developed for cancer patients, but it has been used in samples with various clinical conditions. The BPI rapidly assesses the severity of pain and its impact on functioning in daily life. The pain items are composed of (1) worst pain in last $24 \mathrm{~h}$, (2) least pain in last $24 \mathrm{~h}$, (3) pain on average, and (4) current pain. A numerical rating scale (NRS) was used to measure pain intensity at each assessment, where 0 indicates "no pain" and 10 "the greatest pain possible." The BPI pain score is calculated as the mean of the four scores. The second set of items assess the degree of pain interference in daily activities. The items include relationships with others, enjoyment of life, mood, and sleep, as well as walking, general activity, and working. Cronbach's $\alpha$ was 0.88 for the total score. Scores for the mean BPI-interference range from 0 to 10 , with higher scores indicating higher levels of interference.

PDAS [17] The PDAS is a 20-item tool that assesses the degree of impact of pain-related disability on a person's lifestyle during the past week. Cronbach's $\alpha$ was 0.91 for the total score. Scores for the total PDAS range from 0 to 60 , with higher scores indicating higher levels of pain-related disability.
PCS Pain catastrophizing has been conceived as an exaggerated negative "mental set" brought to bear during actual or anticipated pain experience [22]. The original PCS consists of 13 items, and subjects rate how frequently they have catastrophizing thoughts about pain, with higher scores ( $0-52$ points) indicating a greater degree of catastrophizing. A six-item (items 4, 5, $6,10,11$, and 13) short form of the PCS (PCS-6) was developed in order to facilitate quick screening and to reduce participant burden. In addition, the six-item short form of the PCS was found to be comparable to the original PCS for assessing pain catastrophizing in patients with musculoskeletal pain [18]. Cronbach's $\alpha$ was 0.91 for the total score of the PCS-6. Scores for the total PCS- 6 range from 0 to 24 , with higher scores indicating higher levels of catastrophizing thought.

PSEQ Self-efficacy beliefs in people with chronic pain have been assessed by the strength of a patient's beliefs in their ability to accomplish various activities despite pain [23]. The original PSEQ is an established 10-item measure of pain self-efficacy that is widely used in clinical and research settings. The two-item short form PSEQ (PSEQ-2, using items 5 and 9 in the original PSEQ) appears to be a robust brief measure of pain self-efficacy in people experiencing chronic pain [19]. Cronbach's $\alpha$ was 0.82 for the total PSEQ-2 score. Scores for the total PSEQ-2 range from 0 to 12 , with higher scores indicating higher levels of self-efficacy despite pain.

\section{Physical Function Measures}

Physical function was assessed at the beginning and the end of the program. Physical functional measures included (1) Timed Up and Go test (TUG) [24], (2) two-step test [25], (3) handgrip strength [26], and (4) one-leg standing time (OLST) with eyes open [27].

TUG TUG measures the time it takes a subject to stand up from a chair, walk a distance of $3 \mathrm{~m}$, turn and walk back to the chair, and sit down [24]. All subjects performed two trials, and the better time was used. TUG was originally established as an objective measure of physical 
function in the elderly population. It is also used to assess the risk of falls in older adults [28].

Two-Step Test The two-step test involves the subject starting from a standing posture and moving two steps forward with maximum stride, with caution not to lose balance. If the subject succeeds in holding the final standing position longer than $3 \mathrm{~s}$ without any additional steps, the trial is considered completed. The distance is then standardized by dividing it by the subject's height. The test is performed twice, and the better result is recorded. The value of the two-step test is strongly correlated with maximum walking speed [25], and is well known to be associated with locomotor disabilities among Japanese elderly [29].

Handgrip Strength Handgrip strength was measured bilaterally in a standing position using a Smedley III hand dynamometer. Both sides were tested, and the better value was adopted. Handgrip strength is commonly used as an indicator of overall body strength [26], and it has been shown to be inversely associated with survival [30].

OLST The one-leg standing with eyes open test was tried twice using the preferred leg. We measured the time it took between when the patient started to raise the leg until placing it back down on the floor, and the better time of two trials was adopted [27]. OLST can predict fall risk, especially for the elderly who cannot stand over $10 \mathrm{~s}$ on a single leg [31], and OLST has demonstrated a negative linear relationship with the risk of hip fracture [32].

\section{Multidisciplinary Pain Management Program}

The multidisciplinary program in this study was derived from the CBT-based pain management program described by Nicholas et al. [5]. The participants completed a 3 -h session (13:30 to $16: 30 \mathrm{pm}$ ) on a twice-weekly basis for 4 weeks (total time: $24 \mathrm{~h}$ ). The contents of the sessions are outlined in Table 1. Attention was drawn to specific chapters according to the topics of the day. The orthopedist (T.I.) who studied the multidisciplinary program directed by Professor
Nicholas in Australia was responsible for all lecture and discussion sessions. The subject matter of these sessions is briefly described below.

Models of Chronic Pain This covered the definition of "chronic pain," and differences between acute pain and chronic pain. Although acute pain is normally attenuated along with healing of tissue insults, chronic pain may continue even if tissue insults are repaired. Moreover, psychological problems (e.g. depression and anxiety) are a frequent complication that significantly changes the prognosis and course of chronic pain [33]. Chronic pain can also reduce ADL and quality of life, and may become a burden not only on the patient but also on family members and others involved [34].

Red Flag Signs Pain is fundamentally an alarm system that has evolved to protect us from injury. The alarm symptoms may include compression fractures, malignant tumors, serious infections, or other primary diseases [35]. For example, although vertebral fracture is one of prevalent causes of lower back pain (LBP) among elderly people, it may be overlooked [36]. Most often pain at night or at rest is also considered a red flag for various underlying pathologies [37]. Thus a careful examination is required at least once for pain lasting over 3 months.

Self-Management Strategies Taught Using a CBT Approach for Chronic Pain The most empirically supported methods for teaching chronic pain self-management are based on CBT principles that target changes in both cognition and behaviors [38]. A typical treatment protocol for CBT will involve methods aimed directly at assessing the thoughts associated with pain, the extent of avoidance of unpleasant thoughts and painful experiences, and the consequences of these [38]. Participants learned the CBT model (i.e. relationship between thoughts, emotion, behavior, and physical sensation), and were expected to practice the self-management skills taught between sessions. These included activity pacing, goal 
Table 1 Summary of the self-management program

\begin{tabular}{|c|c|c|}
\hline & Tuesday & Thursday \\
\hline \multirow{7}{*}{$\begin{array}{c}\text { Week } \\
1\end{array}$} & Welcome (introductions) & Practice relax/stretches \\
\hline & Assessment of physical function & Introduce desensitization (meditation) \\
\hline & $\begin{array}{l}\text { Introduce models of chronic pain/Caution to red } \\
\text { flags sign }\end{array}$ & $\begin{array}{l}\text { Discuss self-management strategy for chronic pain based } \\
\text { on CBT approach }\end{array}$ \\
\hline & Discuss goals of pain self-management & Introduce functional exercises \\
\hline & Introduce relax/stretches & Homework assignments \\
\hline & Goal setting & \\
\hline & Homework assignments & \\
\hline \multirow{5}{*}{$\begin{array}{l}\text { Week } \\
2\end{array}$} & Weekend review (of achievements) & Practice stretches/desensitization (meditation) \\
\hline & Practice stretches/desensitization (meditation) & Discuss role of thoughts and thought management for \\
\hline & Discuss pacing and tolerance & pain management \\
\hline & Practice functional exercise & Practice functional exercise \\
\hline & Homework assignments & Homework assignments \\
\hline \multirow{6}{*}{$\begin{array}{l}\text { Week } \\
3\end{array}$} & Weekend review (of achievements) & Practice stretches/desensitization (meditation) \\
\hline & Practice stretches/desensitization (meditation) & Discuss dealing with flare-ups \\
\hline & Discuss central sensitization and chronic pain & Practice functional exercise \\
\hline & mechanism & Homework assignments \\
\hline & Practice functional exercise & \\
\hline & Homework assignments & \\
\hline \multirow{6}{*}{$\begin{array}{l}\text { Week } \\
4\end{array}$} & Weekend review (of achievements) & Practice stretches/desensitization (meditation)/functional \\
\hline & Practice stretches/desensitization (meditation) & exercise \\
\hline & Discuss the use of medication for chronic pain & Home planning for 3 months \\
\hline & management and placebo & Assessment of physical function and questionnaire \\
\hline & Practice functional exercise & Graduation \\
\hline & $\begin{array}{l}\text { Group work and presentation: plan maintenance of } \\
\text { treatment gains }\end{array}$ & \\
\hline
\end{tabular}

Bold indicates items that differ from Nicolas et al. (2013)

setting, and daily relaxation and exercise sessions [39].

Pacing and Tolerance Activity pacing comprises two parts: (1) conserving energy for valued activities, and (2) setting graduated activity quotas to increase the ability to perform activities (tolerance) and reduce disability despite the presence of pain [40].

Goal Setting Goal setting is intended to provide the patient with the motivation to work towards achieving specific goals both during and following the program. Goals may include 
improved health-related quality of life, improved emotional status, and a greater sense of self-efficacy [41]. The participants were asked "to make sure your goals are clear, reachable, and meaningful to you, each goal should meet the SMART criteria (i.e. specific, measurable, achievable, realistic, and time-limited)" [42]. The SMART framework is easy to teach, easy to remember, and has been employed successfully across multiple disciplines, including medical education [43]. The participants were also instructed that the goal should be divided into short- and long-term goals [44].

Desensitization (Meditation) The fear-avoidance model has been one of the most influential behavioral pain models for the past 40 years [45]. One clinical implication of the model is that reducing the threat value of a specific pain should diminish the disability related to that pain. Nicholas et al. studied whether an interoceptive exposure method aimed at reducing pain-avoidance behaviors would add benefit to standard CBT [46]. Although the results indicated that this add-on to CBT did not differ significantly from CBT alone, higher adherence in each group was associated with improvement on measures of pain, pain perception, disability, and depression [46]. Here, we encouraged the participants to think that "(1) the pain is just activity in your nerves, (2) this pain is not acting as a warning signal, and (3) it is just pain and isn't harmful."

Role of Thoughts and Thought Management for Pain Management/Dealing with FlareUps Because unhelpful ways of thinking, like catastrophic thoughts, have been associated with higher pain-related distress [22], it is important for patients to learn to identify such thoughts and to change them to more helpful ways of responding to their pain, especially flares in pain. Thus, the patients were asked to monitor their thoughts in reaction to flares in pain and to identify any that might be unhelpful and risk promoting more distress (e.g. "When will my pain decrease?", "I can't do anything"). Alternative, more helpful responses were discussed, and the patients were encouraged to generate these sorts of thoughts whenever they noticed they were having unhelpful thoughts (e.g. "This pain will subside soon," "I have had this level of pain before, and I know it will subside if I calm myself"). In addition, the patients were encouraged to remind themselves of more constructive thinking (e.g. "What can I do instead of just using more medication or depending on another person?", "What was my goal that I was trying to achieve?") [44].

Central Sensitization and Chronic Pain Mechanism Central sensitization is a condition of the nervous system that is associated with the development and maintenance of chronic pain. The neuroplastic processes may result in gain (sensitization) or loss (desensitization) of function in relation to the incoming nociceptive signals [47]. This discussion was intended to help the patients understand that more injury was not the only possible explanation for their chronic pain, and other, harmless mechanisms were probably involved.

The Use of Medication for Chronic Pain Management and Placebo Placebo effects are positive outcomes that are attributable to the psychosocial context and individual treatment expectations rather than the action of the medication or intervention. The mental events induced by placebo administration can activate analgesic mechanisms that are similar to those activated by drugs [48]. This discussion was intended to help patients understand that even without medication, their pain could be affected by these sorts of mechanisms.

Throughout the program, the nurse and the physical therapist promoted the integration of all session topics in the program. The nurse was responsible for promoting the psychological skills and the physical therapist for the activities/exercises. All participants were encouraged to perform the exercises and skills both during the treatment sessions and at home between sessions. Self-monitoring of homework was also encouraged, and evidence of attempts to practice the exercises and to apply the skills at home was reinforced with praise at each session. Specific skills taught included setting specific, functional (and realistic) goals that were 
meaningful to the participant, activity pacing, arousal reduction using a combination of cognitive challenging and dispassionate exposure, dealing with flare-ups, and structured problemsolving. The program of exercise included stretching and strength training. The exercise program was designated as "high," "moderate," or "low" level depending on the physical functioning of each participant. For example, a participant whose TUG at baseline was less than $6.5 \mathrm{~s}$ in men or less than $7 \mathrm{~s}$ in women was assigned to a "high-level" program, and those with more than $6.5(+2 \mathrm{SD}) \mathrm{s}$ in men and more than $7.0(+2 \mathrm{SD}) \mathrm{s}$ in women were assigned to a "low-level" program, based on the report by Muramoto et al. [49]. Each exercise program included muscle strengthening, balance training, and aerobic exercises. For example, the muscle strengthening exercise program included knee extensor, knee flexor, hip abductor, ankle plantar flexors, and ankle dorsiflexors. Balance training included knee bends, one-leg stand, stepping, etc., for fall prevention [50]. Rubber resistance bands (THERABAND ${ }^{\circledR}$ ) of different magnitudes were used during exercises according to the exercise level and individual capacity. The participants were regularly encouraged to consistently utilize pacing principles in their graded increase in physical activity. Any changes in medications were left to the participants to discuss with their attending doctors. Medication reduction was not a specific goal of the treatment, due to time constraints, and it was felt this was best left to the prescribing doctors.

\section{Statistical Analysis}

All continuous variables were expressed as median (range) or mean (standard deviation [SD]). The normality of the distribution for each measurement was evaluated using the Shapiro-Wilk test for continuous variables. Pain disability (PDAS score) was the primary outcome, and the baseline scores were compared with the 3-month follow-up using the paired $t$ test. Cohen's $d$ was used to evaluate the magnitude of the effect size, calculated by standardized mean difference, with $d$ ranges of $0.2-$ $0.5=$ small effect, $0.5-0.8=$ medium effect, and $d>0.8=$ large effect [51]. One-way repeatedmeasures analysis of variance (ANOVA), followed by Bonferroni post hoc comparisons to control for possible type I errors, was also used to compare the differences in scores of questionnaires across each assessment. The physical function at the beginning and immediately after the program was compared using matched pair Wilcoxon or Student $t$ test, depending on the distribution of the data.

To examine the association between changes in pain disability, psychological or motor functioning during the 4-week program, and changes in outcomes from baseline to final follow-up, correlation coefficients between changes in functional parameters and patientreported outcomes during the program and changes in psychosomatic function between baseline and final follow-up were analyzed using Spearman's test. All statistical analyses were performed using Excel BellCurve ${ }^{\circledR}$ (Social Survey Research Information Co., Ltd.). Differences were considered statistically significant at a level of $p<0.05$.

\section{RESULTS}

The demographic characteristics of the participants are described in Table 2 . Women comprised $81.5 \%$ of the total participants. More than $50 \%$ of the participants had pain in the back or lower back.

The primary outcome, pain disability (PDAS score), among participants was significantly improved at the 3-month follow-up after the program (mean $\pm \mathrm{SD} ; 12.3 \pm 9.0$ ) compared to the baseline $(18.4 \pm 13.2)$, with a medium effect $(d=0.55, p<0.01)$. Also, of the self-report secondary outcomes, all (mean score of BPI-interference, total scores of PCS-6 and PSEQ-2) except participants' pain severity significantly improved immediately after the program compared to baseline, and these effects were maintained over 3 months (Fig. 2). Although mean pain severity was not significantly improved immediately after the program, there was a significant improvement at the 3-month follow-up relative to baseline (Fig. 3). 
Table 2 Participant characteristics at baseline

\begin{tabular}{ll}
\hline Variable & Total sample $(\boldsymbol{n}=\mathbf{2 7})$ \\
\hline Gender (male/female) & $5 / 22$ \\
Age (years) & $73.5(5.7)$ \\
Height $(\mathrm{cm})$ & $155.1(7.8)$ \\
Weight $(\mathrm{kg})$ & $55.3(11.1)$ \\
BMI $\left(\mathrm{kg} / \mathrm{m}^{2}\right)$ & $23.0(4.1)$ \\
Painful area \% (no.) & \\
Neck-head & $14.8(4)$ \\
Upper extremities ${ }^{\mathrm{a}}$ & $22.2(6)$ \\
Lower extremities & \\
Back to lower back & \\
Pain length term $\left(\mathrm{months}^{\mathrm{b}}\right)$ & $107.4(145.8)$ \\
Pain severity $(0-10)$ & $55.6(15)$ \\
Worst & \\
Least & $5.2(2.5)$ \\
Average & $2.4(1.9)$ \\
Current & $4.0(2.0)$ \\
BPI-interference (0-70) & $3.7(2.7)$ \\
PDAS (0-60) & $23.8(14.2)$ \\
PCS-6 (0-24) & $18.4(13.2)$ \\
PSEQ-2 (0-12) & $13.5(6.6)$ \\
\hline Var & $6.8(2.6)$ \\
\hline
\end{tabular}

Value: mean (SD)

BPI Brief Pain Inventory, PDAS Pain Disability Assessment Scale, PCS Pain Catastrophizing Scale 6-item version, PSEQ-2 Pain Self-Efficacy Questionnaire 2-item version

${ }^{a}$ Upper extremities indicates from shoulder to hand, and pain diseases include adhesive capsulitis and osteoarthritis of hand

b Lower extremities indicates from hips to foot, and pain diseases include lumbar spinal canal stenosis and osteoarthritis of the hip, knee and ankle

${ }^{c}$ Pain diseases of back to lower back include degenerative spondylosis, spondylolisthesis, degenerative scoliosis, and spinal canal stenosis

Of the physical function measures, TUG, two-step test, and handgrip strength were significantly improved immediately after the program compared with baseline measures. OLST did not show a significant change from before to after the program (Table 3).

Improvements in the TUG during the 4-week program correlated with the improvements in total scores of BPI-interference, PCS-6, and PSEQ-2 between baseline and final follow-up (Table 4). There was no significant (just) correlation between improvements in the TUG during the 4-week program and improvements in the PDAS score between baseline and final follow-up (Pearson's $r=0.375, p=0.054$ ). In contrast, changes in self-reported function during the 4-week program did not correlate with any changes in those between baseline and final follow-up (Table 4). No adverse events relevant to the program were reported during the programs and follow-up periods. In addition, none of the participants added new analgesic agents or other therapies during the program and follow-up periods.

\section{DISCUSSION}

This study investigated the effect of a CBT-based multidisciplinary pain management program based on the program reported by Nicholas et al. [5] in a sample of elderly Japanese people with chronic pain. The results showed that pain disability, interference of pain in daily activities, pain catastrophizing, and pain self-efficacy amongst the participants improved immediately after the program, and these improvements were maintained over the 3-month follow-up. No adverse events were reported during the program or follow-up periods. Although the current study did not have a control group, a recent report indicates that randomized controlled trials reflect clinical practice when comparing similar populations [52]. According to results from an RCT by Nicholas et al., the effect size $(d)$ of improvement in pain disability at the end of the program and at 1-month and 6-month follow-ups was $0.57,0.60$, and 0.48 respectively, which was a medium effect [6]. In the current study, the improvement in pain disability post-program was similar at the 1-month and 3-month follow- 

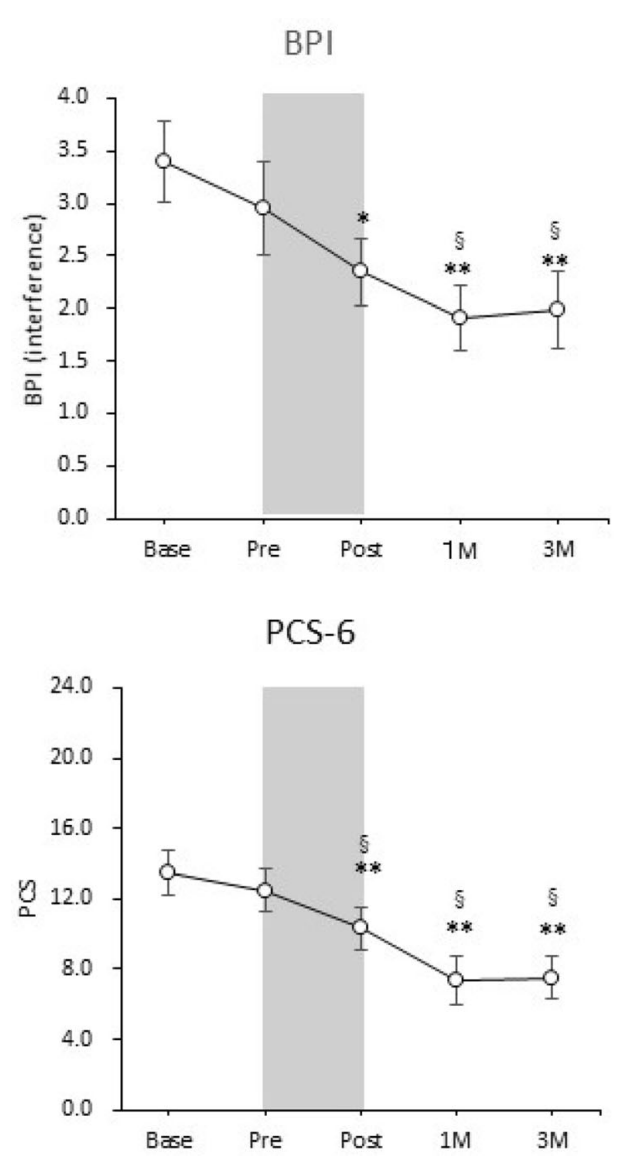

Fig. 2 Changes in scores of each questionnaire. *, ${ }^{* *}$, vs. Base: $p<0.05,0.01 ; \S$, $\$$, vs. Pre: $p<0.05,0.01$; $\dagger$, vs. Post: $p<0.05$. BPI Brief Pain Inventory (term of interference), PDAS Pain Disability Assessment Scale, PCS-6 Pain Catastrophizing Scale 6-item version, PSEQ-2 Pain Self-Efficacy Questionnaire 2-item version, Base

ups (0.54, 0.52, and 0.55, respectively), and also in the medium effect range. These findings suggest that the Japanese multidisciplinary program broadly replicated the results reported by Nicholas et al. [5] and can therefore be considered effective in helping elderly Japanese patients with chronic pain.

Pain behavior is influenced by social, cultural, and psychological factors. For example, it has been reported that Aborigines in Australia did not perceive back pain to be a health problem and consequently did not complain of symptoms, display pain behaviors, or seek treatment [53]. Japan has universal public healthcare: all Japanese citizens are able to have
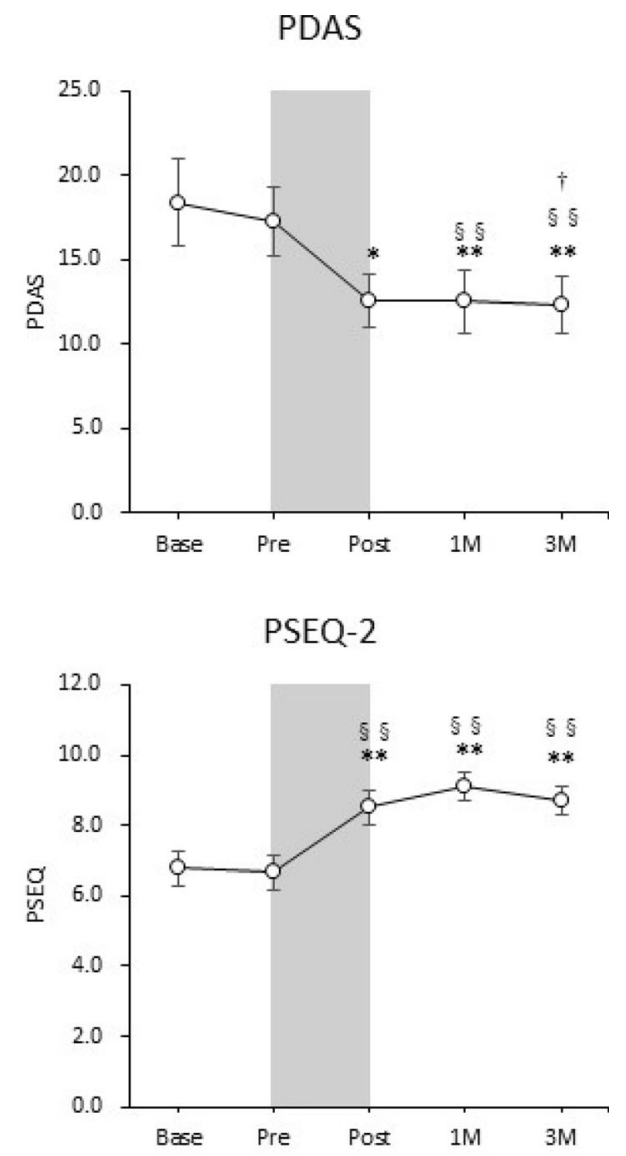

baseline, Pre/Post pre-/post-program, 1M/3M 1-month/ 3-month follow-up. Gray bars indicate a period of intervention

health insurance provided by the state. The Japanese healthcare system does not necessarily distinguish between primary and secondary care, and there is no gatekeeper system [55]; thus people can access very convenient services at affordable prices almost anywhere in the country and receive treatment at a comparatively early stage of any illness [54]. It has been reported that Japan has the largest number of CT and MRI scanners per population; the number of CT scanners per population in Japan is approximately double that in Australia [54]. Frequent testing and visits to doctors have been found to provide little reassurance and instead increase feelings of worry and anxiety among 

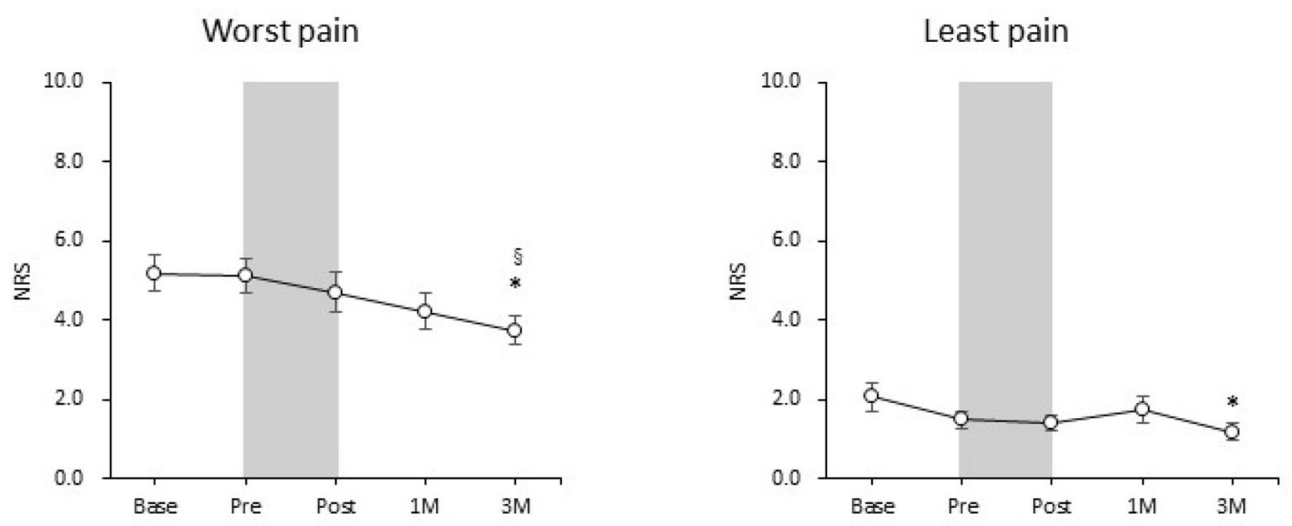

Pain on average

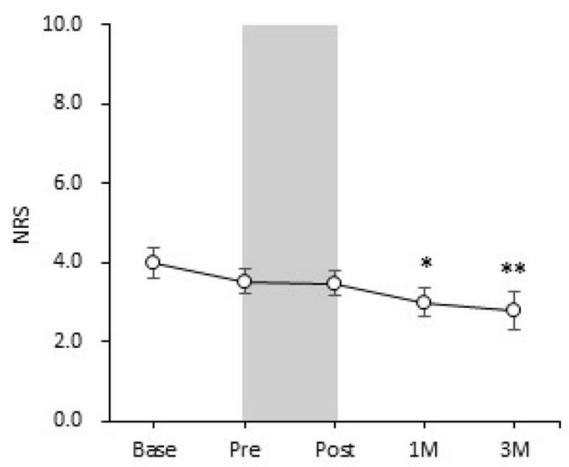

\section{Current pain}

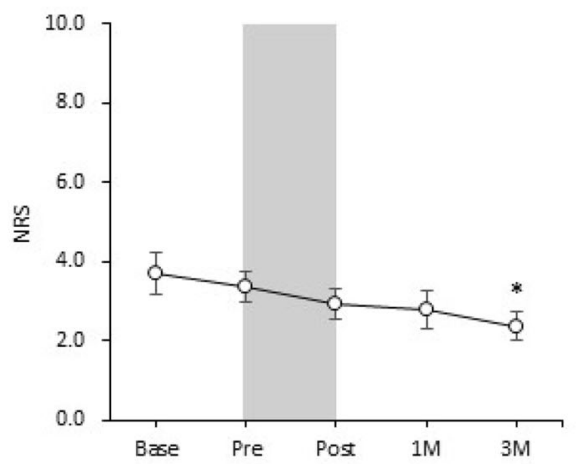

Fig. 3 Changes in pain severity. ${ }^{*}$, ${ }^{*}$, vs. Base: $p<0.05,0.01 ; \S$, vs. Pre: $p<0.05 . N R S$ numerical rating scale, Base baseline, Pre/Post pre-/post-program, $1 M / 3 M$ 1-month/3-month follow-up. Gray bars indicate a period of intervention

Table 3 Mean and standard deviation (SD) of outcome variables of physical function

\begin{tabular}{lccr}
\hline & Pre-program & Post-program & $p$ value \\
\hline TUG $(s)$ & $6.6(5.9-7.4)$ & $6.3(5.6-6.6)$ & 0.003 \\
Two-step test & $1.25(1.13-1.41)$ & $1.36(1.24-1.54)$ & 0.012 \\
Handgrip strength $(\mathrm{kg})$ & & & \\
Right & $22.8(7.16)^{\mathrm{a}}$ & $25.56(6.99)^{\mathrm{a}}$ & $<0.001$ \\
Left & $20.91(7.52)^{\mathrm{a}}$ & $22.44(6.80)^{\mathrm{a}}$ & 0.026 \\
OLST $(\mathrm{s})$ & & & \\
Right & $62(12-120)$ & $50(16-120)$ & 0.509 \\
Left & $71.1(14.8-120)$ & $54.6(26.3-120)$ & 0.349 \\
\hline
\end{tabular}

Value: median (IQR)

TUG Timed Up and Go test, OLST one-leg standing time

${ }^{\mathrm{a}}$ Mean $(\mathrm{SD})$ 
Table 4 The relationship between the immediate effect of 1-month program and the long-term effect of 3 months later

\begin{tabular}{|c|c|c|c|c|c|c|c|c|}
\hline & \multicolumn{8}{|c|}{ Long-term effect (3-month follow-up - baseline) } \\
\hline & \multicolumn{4}{|c|}{$\Delta$ Pain severity } & \multirow[t]{2}{*}{$\Delta$ BPI } & \multirow{2}{*}{$\begin{array}{l}\Delta \\
\text { PDAS }\end{array}$} & \multirow[t]{2}{*}{$\triangle \mathrm{PCS}$} & \multirow{2}{*}{$\begin{array}{l}\Delta \\
\text { PSEQ }\end{array}$} \\
\hline & Worst & Least & Average & Current & & & & \\
\hline \multicolumn{9}{|c|}{ Immediately effect (last day -1 st day) } \\
\hline \multicolumn{9}{|l|}{ Physical function } \\
\hline$\Delta \mathrm{TUG}$ & 0.352 & 0.079 & 0.201 & 0.248 & $0.547^{* *}$ & 0.375 & $0.445^{*}$ & $-0.436^{*}$ \\
\hline$\Delta$ Two-step test & -0.134 & 0.055 & 0.078 & -0.163 & -0.105 & 0.081 & -0.174 & 0.178 \\
\hline $\begin{array}{l}\Delta \text { Handgrip strength (better } \\
\text { side) }\end{array}$ & -0.132 & -0.366 & -0.249 & -0.132 & 0.012 & 0.253 & 0.091 & -0.363 \\
\hline$\Delta$ OLST (better side) & -0.132 & -0.122 & 0.023 & -0.148 & -0.194 & -0.184 & -0.158 & -0.048 \\
\hline \multicolumn{9}{|l|}{ Psychosomatic function } \\
\hline$\Delta \mathrm{BPI}$-interference & 0.305 & 0.296 & 0.141 & 0.088 & 0.180 & -0.143 & -0.103 & 0.135 \\
\hline$\Delta$ PDAS & 0.212 & $0.504^{* *}$ & 0.273 & 0.290 & 0.062 & 0.203 & -0.088 & -0.095 \\
\hline$\Delta$ PCS-6 & $0.483^{*}$ & -0.125 & 0.211 & 0.224 & -0.089 & -0.299 & 0.010 & -0.112 \\
\hline$\Delta$ PSEQ-2 & -0.211 & -0.029 & -0.187 & -0.069 & 0.144 & -0.009 & 0.055 & 0.157 \\
\hline
\end{tabular}

Value: correlation coefficients, ${ }^{*},{ }^{* *}: p<0.05,0.01$

BPI Brief Pain Inventory, PDAS Pain Disability Assessment Scale, PCS Pain Catastrophizing Scale 6-item version, PSEQ Pain Self-Efficacy Questionnaire 2-item version, TUG Timed Up and Go test, OLST one-leg standing time

patients [55]. Nevertheless, psychological treatment of chronic pain in East and Southeast Asia lags behind that in North America and Europe [56].

Although a systematic review showed that CBT-based pain management alone had small positive effects on pain disability and catastrophizing when compared with other therapies such as physical therapy and education [38], the effectiveness of this approach has not been demonstrated in Japan. However, a trial of a multidisciplinary CBT-based program for patients aged $20-80$ years with chronic pain has just recently begun in Japan [10], and this should eventually help to answer this question. The present study has demonstrated that building on an existing and established program rather than trying to develop a new program from scratch may lead to faster accumulation of evidence and dissemination of the value of this approach.
In patients with chronic musculoskeletal pain disorders, an educational strategy addressing neurophysiology and neurobiology of pain is known to have a small positive effect on pain, disability, catastrophizing, and physical performance [57]. Our multidisciplinary program included the same lectures on neurophysiology and neurobiology of pain, but it went further by teaching patients specific pain self-management strategies and an activity upgrade program of exercises, as was done by Nicholas et al. [5]. On the other hand, our modified program included a lecture about caution regarding red flags which differed from the program described by Nicholas et al. [5]. Chronic pain is often regarded as a faulty alarm system (e.g. chronic primary pain [58]), and thus we applied an education approach to reassure subjects that continuing pain signals were not always indicative of harm. However, chronic pain also sometimes implies progressive tissue insults (e.g. osteoarthritis). As elderly people are 
assumed to be biologically more vulnerable than young and middle-aged generations, it is important for them to keep in mind that pain sensation has a role in warning of possible tissue insults.

A recent review of physical activity and exercise for chronic pain reported some favorable effects in reduced pain severity and improved physical function, though these were mostly small to moderate [59]. Our exercise program was determined by individual levels of physical function, and the frequency and the length of exercise time were adjusted by participants themselves, based on advice from physical therapists. In addition, we had weekly reviews (of achievements) and feedback. It is known that interventions such as supervised or individualized exercise therapy and self-management techniques enhance exercise adherence [60].

Interestingly, we found that significant associations between improvements in pain disability (trend of significance), pain self-efficacy, and catastrophizing 3 months after the 4 -week program and decreased time in TUG during the program. Since it was reported that time on TUG correlated with gait speed and ADL among elderly people [24], enhancement in physical performance among those with chronic pain may help to sustain sound mental health as well as physical capacity.

Self-efficacy has been characterized as a protective psychological resource in patients with persistent pain, and a resiliency factor associated with improved functional outcomes in adults with chronic pain $[61,62]$. Importantly, self-efficacy is not a trait, and patients can increase their level of self-efficacy with programs like the one evaluated in the present study. Moreover, high levels of catastrophizing have been shown to be a risk factor for the development of long-term pain and for negative sequelae of pain such as heightened distress, worsening physical disability, and the amplification of pain sensitivity among patients with lower back pain (LBP) and joint pain [61]. As with self-efficacy, our pain self-management program demonstrated that catastrophizing can be reduced in elderly people with chronic pain with the methods employed.
Several limitations of this study need to be mentioned. First, there was no control group. Since we cannot know the natural course of the outcome, our results cannot be interpreted in comparison to a control group. Chronic pain is influenced by different physical, psychological, and social factors [63-65] known as "contextual factors" (CFs). CFs directly impact on the quality of the therapeutic outcomes, with positive CFs (i.e. placebo) reducing pain and negative CFs (i.e. nocebo) increasing pain [65]. As a multidisciplinary pain management program can be regarded as a positive CF for the participants, CFs may have effects on the therapeutic outcomes. However, as an effectiveness trial, our results are consistent with those of studies that have used control groups [5], thereby confirming its effectiveness. Second, our study excluded elderly people with dementia and high care needs for daily living. The number of elderly people who need nursing care is increasing, and $10.7 \%$ of those needing such services in Japan have dementia [1]. Patients with Alzheimer's disease also suffer from chronic pain [66], but clearly, our findings cannot be generalized to this group. Third, this study did not evaluate physical function at 1and 3-month follow-up. Thus, the sustainability of increased physical function is not clear. Fourth, women constituted approximately 80\% of the participants in the current study. Previous research has shown gender differences in description and expression of pain, the use of coping strategies, and the benefit of different treatments $[67,68]$. Therefore, the approach requires further testing with elderly male samples. Finally, this study did not investigate the long-term results beyond 3-month follow up. Therefore, the long-term effects of the imported program need to be evaluated in the future.

\section{CONCLUSION}

This study investigated whether a multidisciplinary pain self-management program modified from that of Nicholas et al. [5] was effective for elderly people with chronic pain in Japan. We found that the program was effective in improving pain disability and achieved similar 
results as those reported by Nicholas et al. This finding has important implications for the development of pain services in communitydwelling elderly Japanese.

\section{ACKNOWLEDGEMENTS}

We would like to acknowledge Mr. Mitsuharu Takahashi, the Seto municipal office, and the Hagiyama community center in Seto city for recruitment of the subjects and providing a place to conduct the program. In addition, we thank the participants of the study.

Funding. This study was supported by research funding of the Department of Orthopaedic Surgery, Aichi Medical University. No Rapid Service Fee was received by the journal for the publication of this article.

Authorship. All named authors meet the International Committee of Medical Journal Editors (ICMJE) criteria for authorship for this article, take responsibility for the integrity of the work as a whole, and have given their approval for this version to be published.

Authorship Contributions. All authors contributed to the following: (1) substantial contributions to conception and design, or acquisition of data, or analysis and interpretation of data; (2) drafting of the article or revising it critically for important intellectual content; (3) final approval of the version to be published.

Disclosures. Tatsunori Ikemoto is a member of the journal's Editorial Board. Yukiko Shiro, Kayo Ikemoto, Kazuhiro Hayashi, Young-Chang Arai, Msataka Deie, Bradley Wood, Lee Beeston, and Michael Nicholas have nothing to disclose.

Compliance with Ethics Guidelines. This study was approved by the Nagoya-Gakuin University Board of Ethics (No. 2017-5). After being informed of the purpose and protocol of the study, participants provided written informed consent before undergoing the study. All procedures performed in studies involving human participants were in accordance with the 1964 Helsinki Declaration and its later amendments or comparable ethical standards.

Data Availability. The datasets generated during and/or analyzed during the current study are available from the corresponding author on reasonable request.

Open Access. This article is licensed under a Creative Commons Attribution-NonCommercial 4.0 International License, which permits any non-commercial use, sharing, adaptation, distribution and reproduction in any medium or format, as long as you give appropriate credit to the original author(s) and the source, provide a link to the Creative Commons licence, and indicate if changes were made. The images or other third party material in this article are included in the article's Creative Commons licence, unless indicated otherwise in a credit line to the material. If material is not included in the article's Creative Commons licence and your intended use is not permitted by statutory regulation or exceeds the permitted use, you will need to obtain permission directly from the copyright holder. To view a copy of this licence, visit http://creativecommons.org/licenses/by$\mathrm{nc} / 4.0 /$.

\section{REFERENCES}

1. Nakamura K. A "super-aged" society and the "locomotive syndrome". J Orthop Sci. 2008;13(1):1-2.

2. Reid MC, Eccleston C, Pillemer K. Management of chronic pain in older adults. BMJ. 2015;13(350): h532.

3. Nakai Y, Makizako H, Kiyama R, Tomioka K, Taniguchi Y, Kubozono T, Takenaka T, Ohishi M. Association between chronic pain and physical frailty in community-dwelling older adults. Int J Environ Res Public Health. 2019;16(8):E1330.

4. Hirase T, Kataoka H, Nakano J, Inokuchi S, Sakamoto J, Okita M. Impact of frailty on chronic pain, activities of daily living and physical activity in community-dwelling older adults: a cross-sectional study. Geriatr Gerontol Int. 2018;18(7):1079-84.

5. Nicholas MK, Asghari A, Blyth FM, Wood BM, Murray R, McCabe R, Brnabic A, Beeston L, Corbett 
M, Sherrington C, Overton S. Self-management intervention for chronic pain in older adults: a randomised controlled trial. Pain. 2013;154(6): 824-35.

6. Nicholas MK, Asghari A, Blyth FM, Wood BM, Murray R, McCabe R, Brnabic A, Beeston L, Corbett $\mathrm{M}$, Sherrington C, Overton S. Long-term outcomes from training in self-management of chronic pain in an elderly population: a randomized controlled trial. Pain. 2017;158(1):86-95.

7. Niknejad B, Bolier R, Henderson CR Jr, Delgado D, Kozlov E, Löckenhoff CE, Reid MC. Association between psychological interventions and chronic pain outcomes in older adults: a systematic review and meta-analysis. JAMA Intern Med. 2018;178(6): 830-9.

8. Cheng JOS, Cheng ST. Effectiveness of physical and cognitive-behavioural intervention programmes for chronic musculoskeletal pain in adults: a systematic review and meta-analysis of randomised controlled trials. PLoS One. 2019;14(10):e0223367.

9. Manchikanti L, Singh V, Kaye AD, Hirsch JA. Lessons for better pain management in the future: learning from the past. Pain Ther. 2020. https://doi. org/10.1007/s40122-020-00170-8.

10. Hosogoshi H, Iwasa K, Fukumori T, Takagishi Y, Takebayashi Y, Adachi T, Oe Y, Tairako Y, Takao Y, Nishie H, Kanie A, Kitahara M, Enomoto K, Ishii H, Shinmei I, Horikoshi M, Shibata M. Pilot study of a basic individualized cognitive behavioral therapy program for chronic pain in Japan. Biopsychosoc Med. 2020;10(14):6.

11. Yoshino A, Okamoto Y, Jinnin R, Takagaki K, Mori A, Yamawaki S. Role of coping with negative emotions in cognitive behavioral therapy for persistent somatoform pain disorder: is it more important than pain catastrophizing? Psychiatry Clin Neurosci. 2019;73(9):560-5.

12. Yoshino A, Okamoto Y, Doi M, Horikoshi M, Oshita K, Nakamura R, Otsuru N, Yoshimura S, Tanaka K, Takagaki K, Jinnin R, Yamashita H, Kawamoto M, Yamawaki S. Effectiveness of group cognitive behavioral therapy for somatoform pain disorder patients in Japan: a preliminary non-case-control study. Psychiatry Clin Neurosci. 2015;69(12): 763-72.

13. Peacock $S$, Patel S. Cultural influences on pain. Rev Pain. 2008;1(2):6-9.

14. American Society of Anesthesiologists Task Force on Chronic Pain Management American Society of Regional Anesthesia and Pain Medicine. Practice guidelines for chronic pain management: an updated report by the American Society of
Anesthesiologists Task Force on Chronic Pain Management and the American Society of Regional Anesthesia and Pain Medicine. Anesthesiology. 2010;112(4):810-33.

15. Brittain E, Lin D. A comparison of intent-to-treat and per-protocol results in antibiotic non-inferiority trials. Stat Med. 2005;24(1):1-10.

16. Cleeland CS, Ryan KM. Pain assessment: global use of the Brief Pain Inventory. Ann Acad Med Singapore. $1994 ; 23(2): 129-38$.

17. Yamashiro K, Arimura T, Iwaki R, Jensen MP, Kubo C, Hosoi M. A multidimensional measure of pain interference: reliability and validity of the pain disability assessment scale. Clin J Pain. 2011;27(4): 338-43.

18. Nishigami T, Mibu A, Tanaka K, Yamashita Y, Watanabe A, Tanabe A. Psychometric properties of the Japanese version of short forms of the Pain Catastrophizing Scale in participants with musculoskeletal pain: a cross-sectional study. J Orthop Sci. 2017;22(2):351-6.

19. Nicholas MK, McGuire BE, Asghari A. A 2-item short form of the Pain Self-efficacy Questionnaire: development and psychometric evaluation of PSEQ-2. J Pain. 2015;16(2):153-63.

20. Inoue $\mathrm{M}$, Ikemoto $\mathrm{T}$, Inoue $\mathrm{S}$, Nakata $\mathrm{M}$, Nishihara M, Arai YP, Miyagawa H, Shimo K, Iida H, Hasegawa T, Wakabayashi T, Sakurai H, Hasegawa Y, Owari K, Hatakeyama N, Ushida T. Analysis of follow-up data from an outpatient pain management program for refractory chronic pain. J Orthop Sci. 2017;22(6): 1132-7.

21. Terwee CB, Bot SD, de Boer MR, van der Windt DA, Knol DL, Dekker J, Bouter LM, de Vet HC. Quality criteria were proposed for measurement properties of health status questionnaires. J Clin Epidemiol. 2007;60(1):34-42.

22. Sullivan MJ, Thorn B, Haythornthwaite JA, Keefe F, Martin M, Bradley LA, Lefebvre JC. Theoretical perspectives on the relation between catastrophizing and pain. Clin J Pain. 2001;17(1):52-64.

23. Nicholas MK. The pain self-efficacy questionnaire: taking pain into account. Eur J Pain. 2007;11(2): 153-63.

24. Podsiadlo D, Richardson S. The timed "Up \& Go": a test of basic functional mobility for frail elderly persons. J Am Geriatr Soc. 1991;39(2):142-8.

25. Muranaga S, Hirano K. Development of a convenient way to predict ability to walk, using a twostep test. J Showa Med Assoc. 2003;63:301-3. 
26. Bohannon RW. Dynamometer measurements of hand-grip strength predict multiple outcomes. Percept Mot Skills. 2001;93(2):323-8.

27. Chang CJ, Chang YS, Yang SW. Using single leg standing time to predict the fall risk in elderly. Conf Proc IEEE Eng Med Biol Soc. 2013;2013:7456-8.

28. Beauchet O, Fantino B, Allali G, Muir SW, MonteroOdasso M, Annweiler C. Timed Up and Go test and risk of falls in older adults: a systematic review. J Nutr Health Aging. 2011;15(10):933-8.

29. Nakamura K, Ogata T. Locomotive syndrome: definition and management. Clin Rev Bone Miner Metab. 2016;14:56-67.

30. Ling $\mathrm{CH}$, Taekema $\mathrm{D}$, de Craen AJ, Gussekloo J, Westendorp RG, Maier AB. Handgrip strength and mortality in the oldest old population: the Leiden 85-plus study. CMAJ. 2010;182(5):429-35.

31. Rikli R, Busch S. Motor performance of women as a function of age and physical activity level. J Gerontol. 1986;41(5):645-9.

32. Lundin $H$, Sääf $M$, Strender LE, Nyren S, Johansson SE, Salminen H. One-leg standing time and hipfracture prediction. Osteoporos Int. 2014;25(4): 1305-11.

33. Tunks ER, Crook J, Weir R. Epidemiology of chronic pain with psychological comorbidity: prevalence, risk, course, and prognosis. Can J Psychiatry. 2008;53(4):224-34.

34. Kowal J, Wilson KG, McWilliams LA, Péloquin K, Duong D. Self-perceived burden in chronic pain: relevance, prevalence, and predictors. Pain. 2012;153(8):1735-41.

35. Foster NE, Anema JR, Cherkin D, Chou R, Cohen SP, Gross DP, Ferreira PH, Fritz JM, Koes BW, Peul W, Turner JA, Maher CG, Lancet Low Back Pain Series Working Group. Prevention and treatment of low back pain: evidence, challenges, and promising directions. Lancet. 2018;391(10137):2368-83.

36. Panda A, Das CJ, Baruah U. Imaging of vertebral fractures. Indian J Endocrinol Metab. 2014;18(3): 295-303.

37. Verhagen AP, Downie A, Popal N, Maher C, Koes BW. Red flags presented in current low back pain guidelines: a review. Eur Spine J. 2016;25(9): 2788-802.

38. Williams AC, Eccleston C, Morley S. Psychological therapies for the management of chronic pain (excluding headache) in adults. Cochrane Database Syst Rev. 2012;11:CD007407.
39. Blake C, Cunningham J, Power CK, Horan S, Spencer $\mathrm{O}$, Fullen BM. The impact of a cognitive behavioral pain management program on sleep in patients with chronic pain: results of a pilot study. Pain Med. 2016;17(2):360-9.

40. Nielson WR, Jensen MP, Karsdorp PA, Vlaeyen JW. A content analysis of activity pacing in chronic pain: what are we measuring and why? Clin J Pain. 2014;30(7):639-45.

41. Levack WM, Weatherall M, Hay-Smith JC, Dean SG, McPherson K, Siegert RJ. Goal setting and strategies to enhance goal pursuit in adult rehabilitation: summary of a Cochrane systematic review and meta-analysis. Eur J Phys Rehabil Med. 2016;52(3): 400-16.

42. Grant H, Dweck CS. Clarifying achievement goals and their impact. J Pers Soc Psychol. 2003;85(3): 541-53.

43. Aghera A, Emery M, Bounds R, Bush C, Stansfield RB, Gillett B, Santen SA. A randomized trial of SMART goal enhanced debriefing after simulation to promote educational actions. West J Emerg Med. 2018;19(1):112-20.

44. Ikemoto T, Miki K, Matsubara T, Wakao N. Psychological treatment strategy for chronic low back pain. Spine Surg Relat Res. 2018;3(3):199-206.

45. Vlaeyen JWS, Crombez G, Linton SJ. The fearavoidance model of pain. Pain. 2016;157(8):1588-9.

46. Nicholas MK, Asghari A, Sharpe L, Brnabic A, Wood BM, Overton S, Tonkin L, de Sousa M, Finniss D, Beeston L, Sutherland A, Corbett M, Brooker C. Cognitive exposure versus avoidance in patients with chronic pain: adherence matters. Eur J Pain. 2014;18(3):424-37.

47. Arendt-Nielsen L, Morlion B, Perrot S, Dahan A, Dickenson A, Kress HG, Wells C, Bouhassira D, Mohr Drewes A. Assessment and manifestation of central sensitisation across different chronic pain conditions. Eur J Pain. 2018;22(2):216-41.

48. Colloca L, Benedetti F. Placebos and painkillers: is mind as real as matter? Nat Rev Neurosci. 2005;6(7): 545-52.

49. Muramoto A, Imagama S, Ito Z, Hirano K, Ishiguro N, Hasegawa Y. Physical performance tests are useful for evaluating and monitoring the severity of locomotive syndrome. J Orthop Sci. 2012;17(6): 782-8.

50. Liu-Ambrose T, Davis JC, Best JR, Dian L, Madden K, Cook W, Hsu CL, Khan KM. Effect of a home-based exercise program on subsequent falls among community-dwelling high-risk older adults after a fall: a 
randomized clinical trial. JAMA. 2019;321(21): 2092-100.

51. Cohen J. Statistical power analysis for the behavioral sciences. Hillsdale: Lawrence Erlbaum Associates; 1988.

52. Maas ET, van Dongen JM, Juch JNS, Groeneweg JG, Kallewaard JW, de Boer MR, Koes B, Verhagen AP, Huygen FJPM, van Tulder MW, Ostelo RWJG. Randomized controlled trials reflected clinical practice when comparing the course of low back pain symptoms in similar populations. J Clin Epidemiol. 2019;116:122-32.

53. Honeyman PT, Jacobs EA. Effects of culture on back pain in Australian aboriginals. Spine (Phila $\mathrm{Pa}$ 1976). 1996;21(7):841-3.

54. Sakamoto H, Rahman M, Nomura S, Okamoto E, Koike S, et al. Japan Health Systems Review. In: Asia Pacific Observatory on Health Systems and Policies. 2018;8(1). http://www.searo.who.int/entity/asia_ pacific_observatory/publications/hits/hit_japan/ en/. Accessed 7 Aug 2020.

55. Barsky JA, Borus JF. Functional somatic syndromes. Ann Intern Med. 1999;130(11):910-21.

56. Yang SY, McCracken LM, Moss-Morris R. Psychological treatments for chronic pain in East and Southeast Asia: a systematic review. Int J Behav Med. 2016;23(4):473-84.

57. Louw A, Diener I, Butler DS, Puentedura EJ. The effect of neuroscience education on pain, disability, anxiety, and stress in chronic musculoskeletal pain. Arch Phys Med Rehabil. 2011;92(12):2041-56.

58. Nicholas M, Vlaeyen JWS, Rief W, Barke A, Aziz Q Benoliel R, Cohen M, Evers S, Giamberardino MA, Goebel A, Korwisi B, Perrot S, Svensson P, Wang SJ, Treede RD, IASP Taskforce for the Classification of Chronic Pain. The IASP classification of chronic pain for ICD-11: chronic primary pain. Pain. $2019 ; 160(1): 28-37$.

59. Geneen LJ, Moore RA, Clarke C, Martin D, Colvin LA, Smith BH. Physical activity and exercise for chronic pain in adults: an overview of Cochrane Reviews. Cochrane Database Syst Rev. 2017;1: CD011279.

60. Jordan JL, Holden MA, Mason EE, Foster NE. Interventions to improve adherence to exercise for chronic musculoskeletal pain in adults. Cochrane Database Syst Rev. 2010;1:CD005956.

61. Edwards RR, Dworkin RH, Sullivan MD, Turk DC, Wasan AD. The role of psychosocial processes in the development and maintenance of chronic pain. J Pain. 2016;17(9 Suppl):T70-92.

62. Stewart DE, Yuen T. A systematic review of resilience in the physically ill. Psychosomatics. 2011;52(3):199-209.

63. Gerdle B, Ghafouri B, Ernberg M, Larsson B. Chronic musculoskeletal pain: review of mechanisms and biochemical biomarkers as assessed by the microdialysis technique. J Pain Res. 2014;12(7): 313-26.

64. Pelletier R, Higgins J, Bourbonnais D. Is neuroplasticity in the central nervous system the missing link to our understanding of chronic musculoskeletal disorders? BMC Musculoskelet Disord. 2015;12(16): 25.

65. Rossettini G, Carlino E, Testa M. Clinical relevance of contextual factors as triggers of placebo and nocebo effects in musculoskeletal pain. BMC Musculoskelet Disord. 2018;19(1):27.

66. Scherder E, Oosterman J, Swaab D, Herr K, Ooms M, Ribbe M, Sergeant J, Pickering G, Benedetti F. Recent developments in pain in dementia. BMJ. 2005;330(7489):461-4.

67. Bernardes SF, Keogh E, Lima ML. Bridging the gap between pain and gender research: a selective literature review. Eur J Pain. 2008;12(4):427-40.

68. Bartley EJ, Fillingim RB. Sex differences in pain: a brief review of clinical and experimental findings. Br J Anaesth. 2013;111(1):52-8. 